\title{
Study on glucose tolerance in pregnancy as a screening test and diagnostic tool for gestational diabetes mellitus
}

\author{
Abdelmarouf Mohieldein $\mathrm{PhD}^{1}$, Abdullatif Taha $\mathrm{MSc}^{2}$, Babekir Alamin $\mathrm{PhD}^{3}$, Abdelraouf A. Abbas $\mathrm{PhD}^{4}$, \\ Osman M. Alsheikh $\mathrm{PhD}^{5}$
}

\begin{abstract}
:
Introduction: GDM is a common medical problem that results from an increased severity of insulin resistance as well as an impairment of compensatory increase in insulin secretion. GDM is associated with a variety of maternal and fetal complications. Controversy surrounds the ideal approach for detecting GDM, and the approaches recommended for screening and diagnosis are largely based on expert opinion.

Material and Method: This study enrolled 51 pregnant women aged between 20 and 39 years old. All women were invited to do fasting and two hours postprandial blood glucose every two weeks, $2 \mathrm{hr}$. $75 \mathrm{~g}$ OGTT every trimester. Plasma glucose measurements were performed with glucose oxidase method using semi-automated spectrophotometer (Biosystems 310).
\end{abstract}

Results and conclusion: Both normal and GDM cases have normal glucose tolerance in early weeks of pregnancy. However, after 24 weeks of gestation progressive increment of hyperglycemia was obviously observed in GDM cases. Suitable cutoffs in diagnosis of GDM in third trimester are $97 \mathrm{mg} / \mathrm{dl}$ for fasting; 174 $\mathrm{mg} / \mathrm{dl}$ for 1 hour; and $141 \mathrm{mg} / \mathrm{dl}$ for 2 hour.

Gestational diabetes mellitus (GDM) is defined as glucose intolerance of variable degrees with onset or first recognition during the present pregnancy ${ }^{1}$. There are both fetal and maternal complications associated with GDM. Fetal complications include macrosomia, neonatal hypoglycemia, perinatal mortality, congenital malformation, hyperbilirubinemia, polycythemia, hypocalcemia, and respiratory distress syndrome ${ }^{2,3}$. Maternal complications include pregnancy induced hypertension, preeclampsia and an increased risk of cesarean delivery ${ }^{4}$.

There has been considerable debate over the appropriate diagnostic criteria for gestational diabetes mellitus ${ }^{5}$. Therefore, understanding the extent of the prevalence of GDM and the associated complications is hindered by the lack of homogeneity in the diagnostic criteria used in previous studies and by changes over time in the recommended diagnostic glucose values ${ }^{6}$.

1. Assist. Prof. Department of Biochemistry, Faculty of Medicine, Omdurman Islamic University.

2. Researcher in Biochemistry. Aloroba Medical Company. Riyadh -KSA.

3. Prof. of Biochemistry. King Saud University. Riyadh. KSA.

4. Assist. Prof. Department of Biochemistry, Faculty of Science and Technology, Omdurman Islamic University.

5. Assoc. Prof. Department of Biochemistry, Faculty of Medicine, International University of Africa.

Correspondence to: Dr. Abdelmarouf H. Mohieldein. P.O.Box 11414 Khartoum 11111, Sudan. Email: mabdelmarouf@hotmail.com
Thus studying of glucose tolerance during the course of pregnancy has been a continuous need in GDM researches. In spite of the real importance of all the view points, our view point that what is the normal glucose tolerance during pregnancy? and who should be said to have abnormal glucose tolerance?

\section{Objectives and aims}

To determine a cutoff point that should be used for the diagnose of GDM.

\section{Material and methods}

This study enrolled 51 pregnant women 40 of them completed the requested questionnaire and blood testing (20 control cases with no gestational diabetes and 20 gestational diabetes cases).

The studied group age was between 20 to 39 years old. All women were attending polyclinics in Aloroba Medical Company (KSA) during the period from December 2004 to April 2006. All volunteers were informed about the nature of the study and respond to a structured questionnaire, and then underwent standardized measurement of weight, height and body mass index (BMI).

All women were invited to do fasting and two hours postprandial blood glucose every two weeks, $2 \mathrm{hr}$. 75g OGTT (oral glucose tolerance test) every trimester, cases with hyperglycemia indicative of diabetes outside pregnancy were excluded. Decisions concerning management of 
hyperglycemia were left to the clinical judgment of the patients' attending obstetricians.

\section{Procedure of measuring blood sugar}

The OGTT used standard procedure ${ }^{7}$. Briefly a $75 \mathrm{~g}$ anhydrous glucose load is administered after 12 to 14 hours fast and then followed by obtained blood fasting, $1 \mathrm{hr}$ and $2 \mathrm{hrs}$ blood samples from an anticubital fossa vein. The collected samples were kept at $4^{0} \mathrm{C}$ in fluoride containing tubes until centrifugation up to $2 \mathrm{hrs}$ later. Plasma glucose measurements were performed with glucose oxidase method ${ }^{8}$ using semi-automated spectrophotometer (Biosystems 310 ). The coefficient of variation being $1.2 \%$.

\section{Measuring gestational age}

Estimation of gestational age was stated by the obstetrician using last menstruation, abdominal examination and ultrasound measurements.

\section{Statistical data processing}

Statistical data processing was carried out with the use of computer program "the Statistical Analysis System - SAS, version 9.1, 2002, SAS Institute Inc ${ }^{9}$. Significant difference was calculated at $p \leq 0.05$. The correlation analysis carried out by calculation of Pearson's coefficient factor.

\section{Characteristics of targeted samples:}

For normal control subjects, the average age being $27.8 \pm 5.1$, body mass index (BMI) was $26.1 \pm 3.3$; for GDM cases the average age being $29.6 \pm 5.0$, , BMI was $28.5 \pm 3.5$ table 1 .

Table 1-: Characteristics of the studied group

\begin{tabular}{|c|c|c|c|}
\hline \multirow{2}{*}{ variable } & \multicolumn{2}{|c|}{ Mean \pm SD } & \multirow{2}{*}{$\begin{array}{c}\mathrm{P} \\
\text { VALUE }\end{array}$} \\
\hline & $\begin{array}{c}\text { normal } \\
(n=20)\end{array}$ & GDM $(n=20)$ & \\
\hline $\begin{array}{l}\text { AGE } \\
(y r)\end{array}$ & $27.8 \pm 5.1$ & $29.6 \pm 5.0$ & 0.2590 \\
\hline $\begin{array}{l}\text { WT } \\
(\mathrm{Kg})\end{array}$ & $69.2 \pm 9.3$ & $73 \pm 10.2$ & 0.2204 \\
\hline $\begin{array}{l}\text { Hight } \\
(\mathrm{cm})\end{array}$ & $162.8 \pm 8.9$ & $159.9 \pm 1.8$ & 0.2457 \\
\hline BMI & $26.1 \pm 3.3$ & $28.49^{*} \pm 3.4$ & 0.0330 \\
\hline
\end{tabular}

$* \mathrm{P} \leq 0.05$.

\section{$\underline{\text { Results }}$}

Fasting blood sugar during pregnancy
The average of fasting blood sugar $(\mathrm{mg} / \mathrm{dl})$ during the period from $2-12$ weeks of gestation ranged between $83.45 \pm 3.27$ and $85.75 \pm 2.97$ for the normal controls; and between $88.3 \pm 4.9$ and $90.3 \pm 5.5$ for the GDM pregnancies. Although it seemed there was tendency to increase in the average of fasting blood sugar in GDM cases, the difference was not significant.

Average fasting blood sugar during the period from $14-24$ weeks of pregnancy ranged between $81.95 \pm 3.05$ and $83.45 \pm 1.96$ for normal cases; and between $88.42 \pm 4.66$ and $94.47 \pm 7.84$ for GDM cases, the difference was significant ( $\mathrm{p} \leq$ 0.001).

During the period from $26-36$ weeks of pregnancy, the average fasting blood sugar ranged between $84.3 \pm 2.56$ and $86.5 \pm 2.52$ for normal subjects and from $96.89 \pm 6.31$ and $99.58 \pm 6.22$ for GDM cases. The difference was significant (p. < 0.0001 ) throughout the third trimester.

The summary of all fasting blood levels sugar for normal and GDM during the course of pregnancy is shown in figure 1.

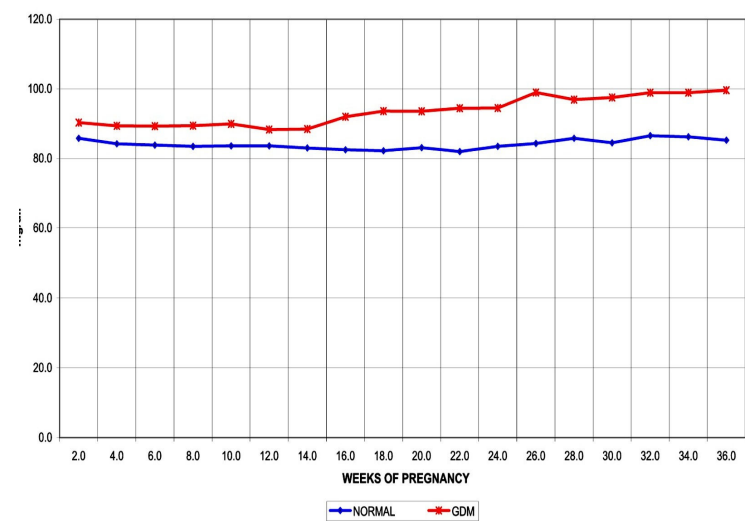

Figure 1. Mean fasting blood sugar for normal and GDM cases during the course of pregnancy.

The 2 hrs. PP blood sugar during pregnancy

The $2 \mathrm{hrs}$ postprandial blood sugar during the period from $2-12$ weeks of gestation ranged between $93.4 \pm 3.84$ and $95.2 \pm 4.1$ for normal cases, and from $99.7 \pm 4.95$ to $105.9 \pm 14.9$ for GDM cases. The difference was significant ( $p<$ $0.05)$

While during the period from $14-24$ weeks of pregnancy the $2 \mathrm{hrs}$ postprandial blood sugar ranged between $91 \pm 5.16$ and $93.9 \pm 3.73$ for normal cases, and between $103.26 \pm 10.44$ and $118.68 \pm 13.2$ for GDM cases. The difference was significant $(\mathrm{p}<0.0001)$.

The $2 \mathrm{hr}$ P.P. blood sugar during 3rd. trimester ranged from $95 \pm 3.29$ and $97.7 \pm 2.83$ for normal cases, and from $123.37 \pm 12.75$ and 
$142.47 \pm 12.65$ for GDM cases, the difference been $30.37-45.42$ and $P$. value $<0.0001$.Fig 2 .

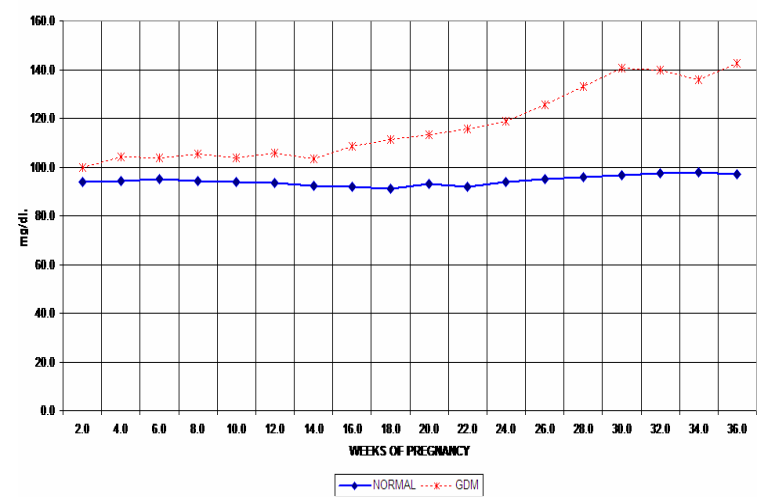

Figure 2. Summary of all $2 \mathrm{hr}$. postprandial blood sugar for normal and GDM during the course of pregnancy.

\section{Oral Glucose Tolerance Test (OGTT) during pregnancy}

OGTT in the first trimester is represented in figure 3, for normal and GDM cases; the difference have been $5 \mathrm{mg} / \mathrm{dl}$ for fasting, 17.7 $\mathrm{mg} / \mathrm{dl}$ for the $1 \mathrm{hr}$ and $9.6 \mathrm{mg} / \mathrm{dl}$ for the two hours values, with considerable $\mathrm{P}$. values sharing significant difference.

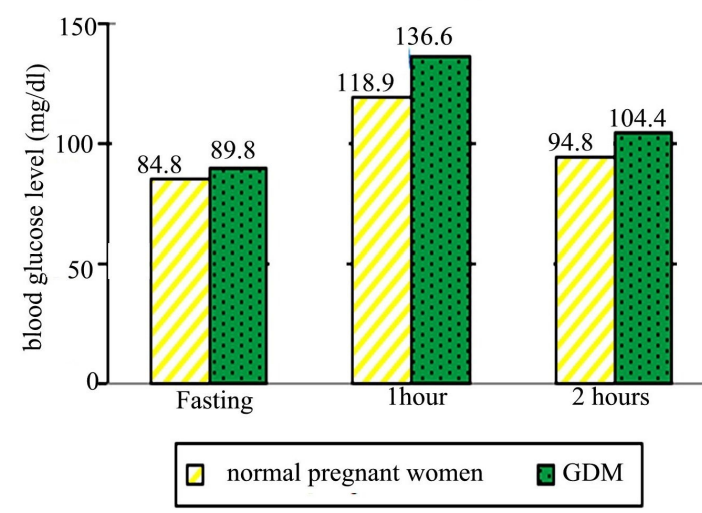

Figure 3. the 75 gram GTT for normal and GD cases during the first trimester (values are expressed in Mean $\pm \mathrm{SD}$ ).

In the second trimester (figure 4), the difference was more obvious and had been 9.75 $\mathrm{mg} / \mathrm{dl}$ for the fasting, 24.4 for the $1 \mathrm{hr}$ and 20.67 for the 2 hrs. P. values been $<0.0001$.

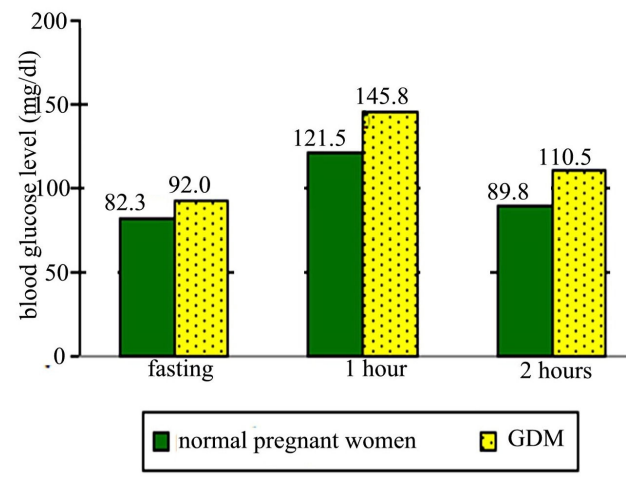

Figure 4. the 75 gram GTT for normal and GD cases during the second trimester (values are expressed in Mean \pm SD).

Figure 5 shows the OGTT for normal and GDM cases in the third trimester and the difference between the two groups was more clear, $12 \mathrm{mg} / \mathrm{dl}$ in the fasting, $44.83 \mathrm{mg} / \mathrm{dl}$ in the 1 $\mathrm{hr}$ and 43.89 in the $2 \mathrm{hrs}$., the P. value been < 0.0001 .

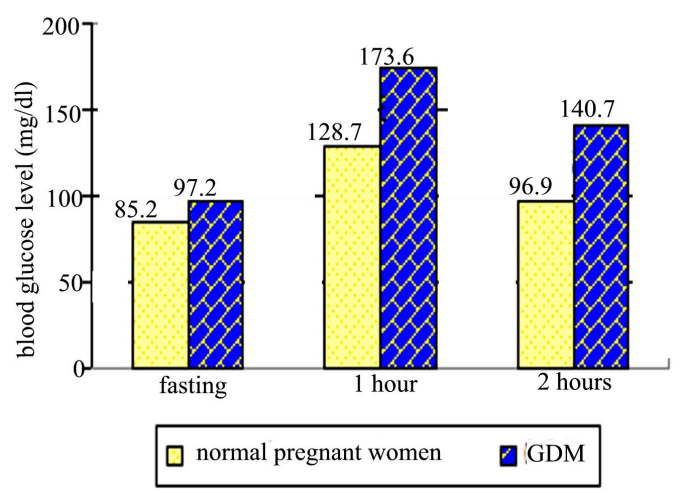

Figure 5. the 75 gram GTT for normal and GD cases during the third trimester (values are expressed in Mean $\pm \mathrm{SD}$ ).

\section{Discussion}

Fasting blood sugar

Although there was significant difference in the average mean of fasting blood sugar level between GDM and normal pregnant women especially in second and third trimester, all the mean values fell within WHO normal reference fasting levels $(126 \mathrm{mg} / \mathrm{dl} \text { WHO } 75 \mathrm{~g} \text { GTT cutoffs })^{10}$.

These results were found to be in contrary with Sacks et $\mathrm{al}^{11}$, who concluded that the fasting plasma glucose assay may perform better than the one hour post-glucose test as a screening test for GDM, and they recommended for a population-based prospective study (Sacks). 
The 2 hrs. postprandial blood glucose

As shown in figure 2; during the first trimester all values fell within normal ranges showing that many GDM cases may display normal glucose tolerance in the fasting as well as in the $2 \mathrm{hrs}$. postprandial tests; this is also true for oral glucose tolerance as shown in figure 3 .

During the second trimester for the 2 hrs. postprandial there also a more significant difference $(\mathrm{p}$ value $<0.0001)$ between the two groups. During the third trimester for the $2 \mathrm{hrs}$. postprandial we observed more clearly the wide variation of glucose tolerance between the two groups $(\mathrm{p}<0.0001)$.

This progressive increment in glucose values since the early weeks of gestation up to the 36 weeks in both cases reflects the suggested insulin resistance of pregnancy and was more clear in GDM cases who were expected to have more insulin resistance, particularly in late weeks of gestation.

So if examination of plasma glucose level is to be done in the $26-36$ weeks of gestation, especially the 2 hrs. p.p, the variation between normal and GDM cases will be more clear and the difference will be high enough to diminish the errors of lab to lab glucose estimation., and there will be yet enough time for treatment to prevent complications suggested to be associated with or as a result of maternal hyperglycemia.

The high values of standard deviation in the postprandial results reflect the increased variation among the same group for postprandial values; this is because of the wide variation in the ingested meal. This is why some providers prefer the OGTT than the $2 \mathrm{hrs}$. postprandial, while those preferring the $2 \mathrm{hrs}$. postprandial argue that this test exactly reflects real life style and not how to tolerate a glucose load.

\section{Oral Glucose Tolerance Test (OGTT)}

Figure 3- show the mean results of the 75g OGTT in the first trimester for normal and GDM cases. These data support that glucose tolerance in both groups tends to be normal during the early weeks of gestation.

The same observation for OGTT found in first trimester is seen in weeks $14-24$, figure 4, where a slightly high difference in the one hour value $(24.4 \mathrm{mg} / \mathrm{dl}$.) between the two groups can be clearly observed.

During the third trimester, Figure 5- the variation is wide enough and the one hour. mean value is $173.6 \pm 12.6 \mathrm{mg} / \mathrm{dl}$ for GDM cases. The difference in this value between the two groups was $44.9 \mathrm{mg} / \mathrm{dl}$ though the fasting readings differed only by $12 \mathrm{mg} / \mathrm{dl}$. Showing that GDM pregnant women differ from normal cases exactly in short term processing of the ingested carbohydrate meal.

In conclusion, according to our data, we can see that:

1. Both normal and GDM cases have normal glucose tolerance in early weeks of pregnancy, with slightly higher plasma glucose values for GDM cases. However, this will not exclude starting hyperglycemia in the first trimester in some of GDM cases.

2. We can also see that the progressive increment of hyperglycemia is more clearly observed in GDM cases particularly after 24 weeks of gestation, with difference values from normal cases higher enough to diminish the variation in readings between different laboratories and between different methodologies estimating blood glucose levels.

3. When we use the 75 gram OGTT in the $3^{\text {rd }}$. trimester to diagnose GDM; the suitable cutoff values seems to be:

$$
\begin{array}{lll}
\text { Fasting : } & 97 \mathrm{mg} / \mathrm{dl} \\
1 \mathrm{hr} . & : & 174 \mathrm{mg} / \mathrm{dl} \\
2 \mathrm{hrs.} & : & 141 \mathrm{mg} / \mathrm{dl}
\end{array}
$$

Which represents the means of GDM cases as shown in the figure 5

\section{Recommendation}

The sample size of this study was small. Further workup using larger sample size is necessary to confirm these results. Despite that we recommend that every pregnant woman should be tested for glucose tolerance in the $24-28$ weeks of gestation.

More restricted cutoff values in GTT during pregnancy should be encouraged so that less cases of GDM will be missed. Also the target glucose levels should be followed with restriction to avoid fetal and maternal complications caused by hyperglycemia during pregnancy.

Our goal from this study was to pay attention for the necessity to revise such reference values of WHO which seems to be high.

\section{References:}

1. Kjos SL, Buchanan TA: Gestational diabetes mellitus. N Engl J Med, 1999; 341:1749-1756.

2. National High Blood Pressure Education. Program Working Group -Report on high blood pressure in pregnancy. Bethesda: NHLBI, NIH; NIH Publication 2000, 00-3029:1-38.

3. Sheffield JS, Butler-Koster EL, Casey BM et al: Maternal diabetes mellitus and infant malformations. Obstet Gynecol , 2002; 925-930.

4. Casey BM, Lucas MJ, McIntire DD et al: Pregnancy outcomes in women with gestational 
diabetes compared with the general obstetric population. Obstet Gynecol 1997; 90:869 -873.

5. American Diabetes Association. Gestational diabetes mellitus. Diabetes Care 2004; 27 Suppl 1:S88-90.

6. Coustan DR: Gestational diabetes. In Diabetes in America. Harris MI, Ed. Bethesda, Maryland, National Institutes of Health, 1995, 703-716.

7. WHO expert committee on diabetes mellitus: Diabetes Mellitus. Geneva, World Health Org., 1985 (Tech. Rep. Ser., no. 727).
8. Trinder P. Determination of glucose in blood using glucose oxidase with an alternative oxygen acceptor. Ann Clin Biochem. 1969; 6:24-27.

9. Statistical Analysis System, (SAS) Institute Inc., Version 9.1 , Cary, NC: SAS Institute Inc., 2002.

10. World Health Organization: Report of a WHO Study Group: Prevention of Diabetes Mellitus. Geneva, World Health Org., 1994 (Tech. Rep. Ser., no. 844).

11. Sacks, et al. Could the Fasting Plasma Glucose Assay Be Used to Screen for Gestational Diabetes? Journal of Reproductive Medicine. 1992; 37(11):907-9. 